\title{
eJRIEPS
}

Ejournal de la recherche sur l'intervention en éducation physique et sport

$47 \mid 2020$

Varia

\section{L'Education Physique et Sportive dans les beaux quartiers. Le cas du lycée Henri IV}

The Physical Education in the neighborhoods. The case of high school Parisian Henri IV

\section{Sarah Pochon et Williams Nuytens}

\section{CpenEdition}

Journals

Édition électronique

URL : http://journals.openedition.org/ejrieps/5173

DOI : $10.4000 /$ ejrieps.5173

ISSN : 2105-0821

Éditeur

ELLIADD

Référence électronique

Sarah Pochon et Williams Nuytens, «L'Education Physique et Sportive dans les beaux quartiers. Le cas du lycée Henri IV », eJRIEPS [En ligne], 47 | 2020, mis en ligne le 01 juillet 2020, consulté le 21 novembre 2020. URL : http://journals.openedition.org/ejrieps/5173; DOI : https://doi.org/10.4000/ ejrieps.5173

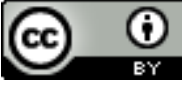

La revue eJRIEPS est mise à disposition selon les termes de la Creative Commons Attribution 4.0 International License. 


\section{L'Education Physique et Sportive dans les beaux quartiers. \\ Le cas du lycée Henri IV.}

Sarah Pochon* \& Williams Nuytens ${ }^{* *}$

* Laboratoire SHERPAS/URePSSS, ULR 7369, Université d'Artois, France.

** Laboratoire SHERPAS/URePSSS, ULR 7369 Université d'Artois, France.

\section{Résumé}

Les travaux consacrés à l'Education Physique et Sportive (EPS) ont peu investi les contextes d'enseignement privilégiés, et encore moins d'excellence. Cet article propose de dresser le portrait d'une EPS qui s'enseigne dans un lieu d'excellence auprès d'élèves très favorisés, en se centrant sur la programmation des activités et les modes organisationnels des leçons. On comprend que malgré une programmation "classique ", les leçons d'EPS d'un lieu d'excellence reposent sur la mesure, la cadence et la vitesse d'apprentissage. On distingue alors une EPS scolarisée, profitant sans doute pleinement de l'influence d'une pédagogisation familiale des activités sportives extrascolaires.

Mots clés: Education Physique et Sportive (EPS), élèves très favorisés, enquête, sociologie, lycée.

The Physical Education in the neighborhoods. The case of high school Parisian Henri IV. Summary

The works in sociology of the Physical Education (PE) poorly studied the privileged contexts of education, and even less excellent. This article suggests drawing up wore it of a PE which teaches in the excellence with very favored pupils, and by centering on the programming of the activities and the organizational modes of the lessons. We understand that the PE of an excellent place does not present particular originality, that the pupils of very favored circles make a success to it of the tasks asked according to a steady rhythm. We distinguish finally a schooled PE, taking advantage doubtless completely of the influence of a family pédagogisation of the extraschool sports activities.

Key words: Physical Education (PE), highly favored students, survey, sociology, high school 


\section{eJRIEPS 47 Juillet 2020}

\section{Introduction}

La recherche en sociologie et en didactique de l'Education Physique et Sportive (EPS) ne se préoccupe pas encore de contextes d'enseignement privilégiés. Pourtant les sociologues de l'École et de l'éducation ont déjà analysé l'autre face de l'effet-établissement. On peut citer par exemple une recherche questionnant l'absence des filles dans les sections scientifiques de l'Ecole Normale Supérieure (Ferrand, Imbert et Marry, 1999), une étude retraçant les logiques qui gouvernent les stratégies d'une élite lycéenne (Dutercq et Daverne, 2009), une autre qui questionne les rapports entre les élèves et quatre grandes écoles françaises (Draelents, 2010) ou celles tournées vers des populations d'élèves aux pratiques ascétiques (Darmon, 2010) et au recrutement social toujours aussi stable dans le temps (De Saint Martin, 2005). On peut comprendre l'intérêt d'étudier d'abord ce qui influence négativement la réussite des élèves (Bressoux, 1994 ; Cousin 1993 ; Duru-Bellat, 2002a) ou ce qui pose problème dans les pratiques professionnelles comme les comportements hors tâches (Bonniot-Paquien, 2009), les inégalités de réussite en termes de sédimentation et de construction scolaire (Vigneron, 2006), les mécanismes d'évaluation intégrant des logiques d'arrangements (Gogérino \& Mnaffakh, 2008), le glissement sportif des contenus enseignés et de ses dérives (Poggi, 2007 ; Sébileau, 2011). Mais l'étude d'un contexte d'excellence pourrait pourtant avoir quelques vertus, notamment pour comprendre autrement les inégalités à l'École. On sait effectivement que les performances scolaires ne dérivent pas seulement du recrutement social des élèves, mais aussi du fonctionnement interne des établissements (Cousin, 1993) et finalement d'une imbrication de variables participant du climat scolaire (Cohen, 2006). Ainsi peut-on supposer qu'une meilleure connaissance de contextes scolaires favorisés permettrait d'accéder autrement à des réalités plus ordinaires ou difficiles. Toutefois il serait naïf de croire à une transposition de conditions d'enseignement, à une reproduction d'une division particulière du travail enseignant d'un milieu à un autre. C'est pourquoi notre propos se limitera à l'examen d'une partie de l'excellence scolaire à travers des normes héritées et fabriquées par l'institution, mais aussi importées par les élèves eux-mêmes afin d'identifier les ingrédients de cette excellence et ce, en les rapportant à l'EPS.

En offrant un environnement éducatif (Duru-Bellat, 2002b) aussi esthétiquement singulier que propice au travail et à l'étude, le quotidien scolaire en milieu d'excellence repose sur un mélange de rigueur et de liberté laissée aux élèves mais aussi sur l'influence d'un milieu familial entrepreneur d'une pédagogisation d'activités extrascolaires (sportives et artistiques) profitables. Comment ces normes institutionnelles et familiales marquent les 


\section{eJRIEPS 47 Juillet 2020}

leçons d'EPS dans leur déroulement et leur organisation ? Sur quelles pratiques reposent les leçons et comment les élèves s'y comportent et apprennent ? Nous répondrons à ces questions après avoir précisé le cadre théorique sur lequel repose notre travail et présenté notre protocole d'enquête. Nous verrons tout d'abord dans quelle mesure ce lieu ressemble à une institution auto-disciplinante. Nous présenterons ensuite les caractéristiques de lycéens pour mieux lire la nature des interactions qui se jouent entre eux et une EPS à la programmation que nous avons choisi de qualifier de « classique » en référence au rapport de Benhaim-Grosse (2007) qui différencie les activités classiques (pratiquées dans plus de $80 \%$ des établissements du second degré) et les activités discriminantes. Enfin, nous donnerons à voir les modes organisationnels particulièrement cadencés des leçons d'EPS.

\section{Le déroulement de la recherche: la démarche inductive dans un contexte d'excellence}

Construite dans les situations de l'établissement et résultant de paramètres qui lui sont extérieurs, l'excellence a été étudiée selon une version ascendante de l'enquête guidée par l'adoption de sensibilités préalablement établies. En ayant choisi de porter notre attention non pas sur « les mondes scolaires disqualifiés » (Payet, 2016, p. 59) mais au contraire sur un monde scolaire prestigieux et performant, il fallait mener les enquêtes dans un établissement dont la renommée s'organise à la fois autour des résultats scolaires des élèves, du recrutement de ces derniers dans les milieux aisés et de son contexte socioculturel privilégié. Le travail de terrain s'est déroulé dans un établissement des «beaux quartiers » parisien (Pinçon-Charlot \& Pinçon, 2007, p.53) : le lycée Henri IV.

\section{1. La socio-ethno-didactique pour cadre d'enquête}

Considérant que «les recherches menées dans les classes témoignent, elles aussi, d'un métissage conceptuel pour travailler le rapport à l'empirie »(Amade-Escot, 2003, p.14), pour mener à bien l'étude et arriver à « saturer » le terrain dans une démarche intensive, nous avons adopté une démarche de recherche reprenant plusieurs cadres théoriques. L'enquête a ainsi rapproché la démarche ethnographique, la sociologie dispositionnaliste et contextualiste, la sociologie des curricula et ce qui peut s'apparenter à une didactique des gestes professionnels des enseignants d'EPS. En empruntant à Poggi et Brière-Guenoun (2014) leur conceptualisation de la socio-didactique, la conjugaison des ancrages théoriques et des instruments conceptuels a abouti au cadre de la socio-ethno-didactique. 


\section{eJRIEPS 47 Juillet 2020}

1.1.1. De la démarche ethnographique à la sociologie dispositionnaliste et contextualiste

La démarche ethnographique est au fondement de l'enquête parce qu'elle permet de procéder par étapes pour situer la discipline à l'intérieur de son contexte. Plutôt que de décrire et analyser immédiatement les gestes professionnels des enseignants, c'est progressivement que nous nous sommes rapprochés des leçons de la discipline. En effet, « il devient banal de rappeler qu'une activité de transmission-appropriation de savoirs est toujours socialement située » (Poggi, 2012, p. 133). On sait que les pratiques enseignantes sont en prise avec le contexte dans lequel elles sont mises en œuvre. Les contextes peuvent être prégnants et peser sur les pratiques jusqu'à influencer la leçon dans ses contenus (le curriculum transmis). Aussi, pour décrire et analyser les pratiques enseignantes à la lumière de ces variables contextuelles, notre priorité devait situer la discipline dans ses conditions sociales de production en interrogeant le contexte d'enseignement, en dressant la sociologie des élèves et des enseignants afin de renseigner l'ailleurs didactique (Sensevy \& Mercier, 2007). Nous avons fait de l'ethnographie scolaire au sens de Michèle Guigue pour qui « l'usage de l'ethnographie met l'accent, d'une part, sur la collecte de données au plus près des acteurs, de leurs situations et des contextes dans lesquels ils agissent et interagissent et, d'autre part, pour valoriser des interprétations contextualisées plutôt que des théories qui surplombent, parfois de très haut, les réalités et les expériences sociales quotidiennes » (Guigue, 2014, p. 10). Les contraintes qui pèsent sur les enseignants sont d'ordre institutionnel bien sûr car les enseignants sont guidés par des programmes mais on constate aussi que des "déterminants externes liés au profil socio culturel des élèves constituent à la fois des ressources et des contraintes pour l'action »(Poggi, 2018, p. 86). En considérant l'interaction comme le produit de l'actualisation de dispositions incorporées mais également comme le produit d'ajustements nécessaires (imposés par les contraintes de la situation) susceptibles d'engendrer des transformations durables de l'habitus, la sociologie dispositionnaliste est en ce sens « [...] attentive aux différentes dispositions qui ont pu façonner les individus» (Zolesio, 2010). On comprend ainsi que la sociologie dispositionnaliste met en avant le fait que "l'acteur est doté d'un passé incorporé qui contraint, à son insu, le présent et, dans le cas qui nous intéresse, la situation d'interaction en classe " (Poggi, 2012, p. 41). Mais l'habitus permet aussi de s'adapter et de faire preuve de créativité lorsque l'acteur fait face à des " décalages, discordances et ratés 》 (Bourdieu, 1997, p. 189). Ces considérations théoriques à propos des capacités d'adaptation des enseignants mais aussi des élèves, nous ont conduit à la mobilisation des cadres de la 


\section{eJRIEPS 47 Juillet 2020}

sociologie dispositionnaliste et contextualiste de Lahire (1998). Dans cette perspective, nous pouvons effectivement envisager la situation comme le lieu d'une rencontre entre un ensemble de dispositions incorporées et un contexte présent de l'action offrant des occasions d'expression, de neutralisation ou encore des transformations de ces dispositions.

1.1.2. La sociologie du curriculum et l'analyse didactique des gestes professionnels

Dans notre travail, la sociologie du curriculum s'associe aux cadres de la sociologie dispositionnaliste et contextualiste pour comprendre et analyser la co-construction des savoirs mises en œuvre dans les leçons d'EPS au lycée Henri IV. Parmi tout ce qui est potentiellement transmissible, les savoirs véhiculés par l'enseignement résultent d'une construction sociale : l'institution, les enseignants font des choix dans ce qu'il y a à enseigner. Or, si comme le montrent les recherches les contenus, les savoirs transmis aux élèves diffèrent selon les contextes d'enseignement, nous avons souhaité étudier le contenu de ces leçons d'EPS mises en œuvre en contexte favorisé afin de comprendre les choix opérés en matière de sélection curriculaire par les enseignants.

Si les cadres de la sociologie du curriculum permettent d'interroger la nature des contenus transmis et les objets culturels mis à l'étude, l'approche didactique nous a permis quant à elle, de questionner les modalités de leurs transmissions. Autrement dit, en nous intéressant au « quoi enseigner ? », nous nous sommes intéressés au «comment enseigner ? en appréhendant les gestes professionnels des enseignants. À la notion assez large de pratiques enseignantes nous avons en effet substitué celle de « gestes professionnels » qui désigne des actes concrets consistant à conduire l'étude d'un sujet en position d'élève dans une institution donnée (Chevallard, 1999). Toute situation didactique peut alors être considérée comme l'ensemble complexe des relations entre le milieu didactique et les actions du professeur et des élèves en direction des objets de savoir devant être enseignés et appris (Amade-Escot, Verscheure et Devos, 2002). Ainsi, pour renseigner les dimensions spécifiques de l'activité enseignante du point de vue didactique, nous avons mobilisé les descripteurs de l'Action Conjointe en Didactique (Sensevy \& Mercier, 2007) et avons découpé les leçons dans les plans mésogénétique, chronogénétique et topogénétique afin d'examiner ce qui est de l'ordre de la définition, de la dévolution, de la régulation et de l'institutionnalisation. L'analyse de ces gestes, constitutifs des modalités courantes de 


\section{eJRIEPS 47 Juillet 2020}

construction du savoir dans la classe (Sensevy, 2002), nous a permis d'accéder à la nature des savoirs transmis en classe.

Donc, si notre travail s'intéresse aux gestes professionnels dans leur dimension didactique ainsi qu'à la question des savoirs enseignés, nous n'avons négligé ni les conditions locales et le contexte social dans lesquels sont mises en œuvre les leçons d'EPS, ni les déterminants sociaux qui pèsent sur les acteurs de la discipline, ni enfin, la capacité d'adaptation des enseignants aux contraintes qui s'exercent sur les situations didactiques. C'est bien la combinaison de ces cadres théoriques et disciplinaires qui autorise la compréhension des choix opérés par ces enseignants en matière de « culture corporelle scolaire » (Poggi, 2012, p. 39) à travers la programmation d'activités physiques sportives et artistiques (APSA) établie et les manières d'organiser les leçons.

\subsection{Le protocole de recueil de données: des techniques d'investigation plurielles}

Notre enquête repose également sur l'usage de plusieurs techniques de recherche. Nos immersions, passations de questionnaires et entretiens compréhensifs ont été menés en considérant le poids de dispositions sociales, des variables de contexte et d'interactions. Cette posture intégrant l'adéquation entre des expériences d'acteurs et des contraintes de l'action s'inscrit dans l'interactionnisme de l'Ecole de Chicago. Nous faisons référence sur ce point aux concepts et procédures développés dans Strauss (1992), ainsi qu'à des applications dans le contexte scolaire comme dans Van Zanten (2001) et Paty (1981).

\subsubsection{Les observations « flottantes » du contexte}

Avant d'entrer dans la classe, il a fallu parcourir l'établissement. Les premières rencontres avec l'excellence débutent par des observations sans but précis (Pétonnet, 1982) dans et autour du lycée, ensuite par des discussions avec le proviseur du lycée et les membres de l'équipe EPS ; nous y avons cherché le mandat pour enquêter. Une première immersion s'est déroulée au début du mois d'octobre 2014, durant une semaine et chaque jour de $8 \mathrm{~h}$ à 17h30. II fallait découvrir le lycée mais à distance des leçons d'EPS, discuter avec des enseignants lors des intercours et échanger avec d'autres membres du lycée (deux documentalistes par exemple). Le travail a nécessité une présence conséquente, une période d'immersion longue et répétée sur le terrain pour favoriser l'intégration et l'empathie, développer des relations avec les enquêtés. Ces premiers temps d'observation ont conduit à la collecte de «données, de documents et informations, et de réflexions théoriques » 


\section{eJRIEPS 47 Juillet 2020}

(Copans, 1966, p. 120). Nous avons essayé de privilégier un aller-retour constant entre description, comparaison et catégorisation (Jacobs, 2016, p. 100) : la conception des grilles d'observation et de lectures des situations d'enseignement a donc reposé sur ces va-etvient recommandés par les spécialistes de la théorisation ancrée (Glaser \& Strauss, 2010), selon une méthode d'interaction circulaire entre la collecte et l'analyse de données appelée aussi «emergent-fit». Trois mois se sont écoulés avant une nouvelle immersion d'une semaine, précédée en décembre d'une visite guidée du lycée réalisée par un historien. Dans notre journal de terrain, chaque jour nous faisions la description de tel ou tel endroit du lycée : le cloître et ses jardins, la cour du méridien, la bibliothèque des prépas, la salle des professeurs...La prise de note, parfois sur le vif, parfois après coup pour éviter un comportement suspect, nous a aussi permis de questionner les axes de recherches et les perspectives de travail, les méthodes d'observation et le protocole de recueil de données « au moment même de l'enquête en réorganisant les données dans un corpus »(Céfaï, 2003, p. 594).

1.2.2. Les observations in situ des leçons d'EPS au collège et au lycée et la construction d'indicateurs de référence

La deuxième immersion (une semaine en janvier 2015) a permis de voir l'EPS en train de se faire, de la sixième à la terminale (l'établissement est une cité scolaire) et d'orienter l'enquête en direction des lycéens. Le lycée n'a en effet rien à voir avec ce qui se joue au collège, de sorte que l'effet d'âge semble avoir son importance y compris pour des élèves de milieux sociaux privilégiés. Au lycée l'EPS s'ordonne : l'écoute et l'attention des élèves se portent sur le professeur, leur mise en tâches est immédiate une fois la consigne donnée, le bruit et l'agitation font place aux discussions à voix basses, l'autonomie et l'application d'une méthodologie de travail règnent. Déjà là, des indices d'un contexte privilégié sautent aux yeux de l'observateur: les leçons sont organisées et construites, les élèves se disciplinent et écoutent au premier signe de régulation professorale, l'autorité de l'adulte n'est pas remise en cause, les élèves restent motivés et s'engagent dans les pratiques de sorte que les arrêts durant les tâches n'existent pas.

\section{2. 3. Les observations directes de trois cycles d'EPS au lycée}

La découverte et l'exploration ont donc précédé des périodes de suivis de classes (seconde, première et terminale), durant un cycle d'enseignement. Les observations se sont déroulées ainsi chaque jeudi de la mi-mars à la fin du mois de mai de l'année 2015. Le choix de ces 


\section{eJRIEPS 47 Juillet 2020}

classes résultait d'un souci pratique et d'une proximité construite avec les trois enseignants au cours de l'enquête. Le jeudi était idéal dans la mesure où il revêtait cet aspect pratique : trois niveaux de classe avaient cours et à des moments différents de la journée et nous connaissions les enseignants. Nous avons ainsi observé la classe de terminale en tennis de table sous la responsabilité de Luc à 8h30, la classe de seconde en tennis de table sous la responsabilité de Monique à 10 h30 (un peu plus tard dans l'année) et la classe de première en acrosport sous la responsabilité de Valérie à 15h30. Conformément à la vision inductive de l'enquête, la grille d'observation a été construite puis réélaborée, enrichie à plusieurs reprises, à partir d'observables précis identifiés au fur et à mesure des premières séances afin de saisir au mieux les aspects didactiques du travail enseignant.

1.2.4. Les techniques d'investigations complémentaires: la passation de questionnaires et les entretiens compréhensifs

Pour éprouver ce monde scolaire de l'excellence, c'est donc « la combinaison d'une longue temporalité et d'une diversité de lieux d'observation » qui a donné de « l'ampleur à [notre] terrain de recherche » (Chartier, 2016, p. 85). En prenant le parti d'investiguer un unique terrain d'enquête, nous avons facilité le contrôle de techniques de recherche et d'outils théoriques variés. A côté de nos immersions nous avons administré directement des questionnaires ROSL qui visaient à caractériser le lieu de Résidence, l'Origine Sociale et les Loisirs des lycéens. La passation directe d'une seconde vague de questionnaires en cours d'enquêtes visait à apprécier le sens donné par les élèves à l'EPS comme discipline, du point de vue des apprentissages (ces dernières datas ne sont pas traitées dans le présent article). Ainsi 631 questionnaires ont été traités sur un total de 857 lycéens. Toutes les terminales et les secondes ont pu être touchées (18 classes) contre six des sept classes des 1 ères (en raison de nombreuses sorties scolaires organisées dans un cas). Dans notre échantillon on notera enfin que $42 \%$ des répondants sont des lycéens de la terminale (35\% de la seconde et le reste de la première), et que la série $S$ est la plus représentée (deux fois plus que de lycéens des séries L et ES réunies). Nous avons en parallèle, réalisé des entretiens informels (avec deux documentalistes, des élèves, une infirmière scolaire, des enseignants) et treize entretiens formels (sept avec les enseignants d'EPS volontaires, trois avec des enseignants de physique et de sciences de la vie et de la terre, un avec le proviseur de l'établissement et la proviseure-adjointe en poste au moment de l'enquête, un avec la proviseure nouvellement nommée à la rentrée 2016). Environ huit heures d'enregistrement ont été exploitées pour alimenter cet article. Dans la mesure où l'analyse 


\section{eJRIEPS 47 Juillet 2020}

de l'activité enseignante pose la question de l'articulation du « visible » et de « l'invisible » (Félix, Amigues et Espinassy, 2014, p. 52), il fallait conduire des entretiens pour obtenir de quoi examiner le curriculum déclaré des enseignants (ce qu'ils disent faire, ce qui leur paraît prioritaire d'être enseigné) et le curriculum réel c'est-à-dire des objets de savoirs réellement transmis à l'intérieur des dispositifs d'apprentissages.

\subsection{Le traitement des données}

Nous avons analysé les données recueillies pour les comparer aux travaux consacrés à l'EPS dans les milieux difficiles, à chaque moment de rupture avec le terrain. L'analyse commence en effet sitôt les premiers matériaux assemblés et se poursuit tout au long du processus de recherche. Nous avons ainsi engagé une analyse systématique dès le début des observations de terrain. Dans cette sociologie qualitative, c'est en quelque sorte « le processus de recherche lui-même qui [nous a guidé] dans l'examen de toutes les pistes prometteuses de compréhension » (Céfaï, 2003, p. 366).

\section{Résultats}

\subsection{Un lieu de vie et une institution auto-disciplinante}

Avec $100 \%$ de réussite au baccalauréat et plus de $98 \%$ de mentions obtenues chaque année depuis 2012, une forte représentation de la filière scientifique (en 2014-2015, 67 \% des élèves de seconde s'orientent vers une première scientifique, contre $11 \%$ en première littéraire et $20,5 \%$ vers une filière économique et sociale), considérée dans le secondaire comme la «filière d'élite» (Cacouault-Bitaud \& Oeuvrad, 2009, p. 30), une richesse d'options diversifiées (on trouve au lycée Henri IV l'enseignement de l'hébreu, du russe, du chinois et de l'italien en plus de l'anglais, de l'espagnol et de l'allemand) et des Classes Préparatoires aux Grandes Ecoles (CPGE) les plus prestigieuses, le lycée Henri IV de Paris se classe en tête des palmarès des meilleurs lycées français. En ce lieu les bâtiments n'ont rien de misérable comme dans le Lycée Professionnel Pablo Néruda de Dubet par exemple (Dubet, 1991), et les élèves studieux des bibliothèques ressemblent à ces étudiants ayant pleinement intériorisé une mise au pas ascétique (Darmon, 2013). Le silence, l'ascétisme et l'absence de surveillants ne sont peut-être que des apparences, des fétiches du prestige comme une marque distinctive qui séparait les membres des élites de la France d'Ancien Régime (Elias, 1969). Mais nous ne le croyons pas. 


\section{eJRIEPS 47 Juillet 2020}

Les conditions écologiques de la construction de l'excellence scolaire semblent reposer sur des normes héritées mais aussi fabriquées par l'institution scolaire elle-même. Les effets de contexte qui se cumulent aux abords et à l'intérieur du lycée Henri IV imprègnent les élèves : l'architecture, le patrimoine hérité. Si on s'intéresse à la géographie et à l'histoire du 5 e arrondissement où se situe le lycée, aussi appelé «quartiers des écoles », on constate la concentration des établissements liés à l'institution universitaire : collèges et vestiges de collèges, établissements religieux, facultés. Si on fait le tour de la cité scolaire en l'observant du dehors, on voit bien que le lycée Henri IV est construit dans la pierre. Son héritage historique est d'ailleurs ce qui caractérise les établissements de la capitale. Dans le cas de l'île-de-France, un collectif de chercheurs (Rhein, Le Pape et Grosbras, 1999) a effectivement montré que « les établissements d'enseignement général se situent dans des espaces urbains centraux et socialement favorisés, d'autant plus réputés qu'ils sont anciens, tandis que les établissements polyvalents, techniques et professionnels, plus récents, sont implantés dans des espaces périphériques, de composition plus populaire » (François \& Poupeau, 2008, p. 94). Le lycée hérite donc de son histoire, ce mélange de style architecturaux, entre art gothique et art roman, entre conservation d'espaces médiévaux et décors datant de l'époque baroque, lui conférant du charme et du cachet, en fait un certain esthétisme. Avant d'être cette cité scolaire du 23 rue Clovis, le lycée est une abbaye fondée en 502 par Clovis et Clothilde.

Or, on sait que les dispositions spatiales et matérielles influencent de manière essentielle la perception qu'ont les élèves des établissements. Dans les collèges étudiés par Paty (1981), les dégradations des collèges sont souvent « un exutoire à l'hostilité envers le collège et les professeurs » (Paty, 1981 cité par Dufrasne, 1982, p. 75). On peut imaginer à l'inverse, que l'environnement interne et externe du lycée, vont imposer le respect du lieu et prédisposer les élèves au travail, à une attitude, de contrôle de soi, sorte d'ascétisme juvénile (Darmon, 2010, p. 49), ce qui ne serait donc en rien contradictoire avec le lieu qu'était originellement le lycée. Certaines particularités de l'établissement renforcent d'ailleurs une certaine atmosphère de travail : le lycée ferme ses portes à minuit en étant un véritable lieu de vie ; ouvert sur l'extérieur, il accueille des conférenciers, d'anciens élèves, des associations, des hommes politiques. La bibliothèque est ouverte le dimanche et le reste jusqu'à 22h30 durant les vacances. Si le lycée Henri IV est l'héritier d'un contexte d'excellence, il fabrique aussi des normes devant influencer les élèves dans leur ambition scolaire et leur rapport au travail. Tandis que le quotidien des élèves ne semble pas encadré ou surveillé (au lycée, il n'y a pas de surveillant et les élèves n'ont pas de carnet de 


\section{eJRIEPS 47 Juillet 2020}

correspondance), le parcours scolaire des élèves lui, est balisé. Bien sûr, la dialectique entre les valeurs de rigueur et de liberté existe dans les autres établissements du second degré mais la différence se joue dans l'acceptation par tous les élèves de ces valeurs renvoyant à l'ordre, au respect, à la discipline. Tandis que dans des collèges par exemple, à recrutement populaire, la qualité de vie est moins bonne, la discipline moins assurée, la clarté des règles est moins nette, l'indiscipline plus répandue, les différentes formes de déviance scolaire fréquentes (retards, absentéisme, bavardage, etc.) (Meuret, 1995 ; DuruBellat, 2002). Ici, la culture et les normes de conduite des élèves correspondent aux attentes de l'institution (Duru-Bellat, ibid), si bien que ce qui est discipliné dans ce contexte, ce n'est pas tant les comportements ou les règles de conduite à tenir que le rapport à l'effort et au travail. Les lycéens sont à l'image des étudiants des classes prépas : "il s'agit d'une population qu'il ne s'agit pas tant de faire obéir que de faire travailler » (Darmon, 2013, p. 51). Le champ lexical repéré dans les discours du proviseur est celui du travail intellectuel et de l'autodiscipline : les élèves doivent fournir des efforts, être rigoureux et employer des méthodes. C'est une obéissance de travail à laquelle ils doivent répondre en étant contraints d'obtenir les meilleurs résultats. Cette exigence de conduite et cette injonction au travail se formalisent dans la lettre que le proviseur adresse personnellement aux élèves de seconde lors de leur rentrée scolaire : il y est question de « ténacité », « d'apprentissages de la rigueur et des méthodes de travail ». Pour renforcer cette autodiscipline, le proviseur utilise au cours de l'année scolaire des discours, des événements, des cérémonies, la remise des bulletins. C'est à ces occasions que les discours prononcés mobilisent divers outils de « mise au travail» (Darmon, 2013, p. 37). Les enseignants d'EPS, lorsque nous les interrogeons, font allusion à ces multiples interventions qui récompensent les meilleurs élèves et peuvent aussi être destinées à ceux qui rencontrent des difficultés (remise des bulletins directement en passant dans les classes de seconde, visites de la tour Clovis, convocations dans le bureau du proviseur pour dialoguer sur les éventuelles difficultés rencontrées...). Ce lycée s'apparente ainsi à l'institution enveloppante définie par Darmon (2013) : « à la fois totale, disciplinaire, protectrice et productrice » (p. 84). C'est une institution qui surveille la productivité des élèves en les mettant au travail et sous pression. Ce lieu balise le parcours de formation des élèves d'examens et de contrôles. Cependant dans la mesure où elle cherche prioritairement à auto-discipliner les élèves dans un cadre de liberté et d'autonomie, nous avons choisi de la caractériser comme une institution autodisciplinante car ce qui y est discipliné concerne moins les comportements ou les règles de conduite et d'obéissance que le rapport à l'effort, au travail (Darmon, 2013) et au temps. 


\section{eJRIEPS 47 Juillet 2020}

L'institution inscrit les élèves dans un rapport au temps typique des élites, qui doit être optimisé, maximisé. Les étudiants des Classes Préparatoires aux Grandes Écoles apprennent en effet à faire du temps la mesure de toute chose (Darmon, 2013, p. 153). On sait à ce propos, que les prépas font « de la vitesse de compréhension et d'exécution une condition de la survie » (Bourdieu, 1989, p. 39 cité par Darmon, 2013, p. 154). Si l'excellence scolaire se mesure dans le rapport au temps, l'excellence en EPS va également reposer sur la vitesse des apprentissages comme si la vitesse était « la forme ultime de l'excellence. Comprendre vite, faire vite »(Darmon, 2013, p. 153).

On voit ici comment l'institution scolaire exerce une pression sur chaque élève qui sait qu'il « fabrique son destin social à l'école, de note en moyenne, de moyenne en passage ou en orientation » et que «la totalité du parcours est dorénavant sous l'emprise d'une très forte injonction à la réussite » (Barrère, 2002, p. 5). Les élèves se retrouvent en quelque sorte pris dans le piège scolaire (Berthelot, 1983). Ils se sont engagés dans des études longues et ambitieuses, et ont le devoir de réussir. L'indépendance des élèves se conjugue à l'exigence de travail et d'autodiscipline repérée dans le discours du proviseur : « Vous devez donc vous prendre davantage en charge, travailler très régulièrement et bien vous organiser, c'est-à-dire devenir très autonome et méthodique, être pleinement responsable et persuadé que rien ne s'obtient sans effort. Comme tous les élèves qui entrent au lycée Henri IV vous avez fait le choix d'un projet d'études ambitieux. C'est bien volontiers que nous nous attacherons à vous aider à le réaliser dans les meilleures conditions. Cependant, cela nécessitera de votre part des efforts et de la ténacité. Vous allez découvrir ce qu'est l'apprentissage de la rigueur et des méthodes de travail » (Extraits de la lettre de rentrée 2014-2015 du proviseur, adressée personnellement à chaque nouvel élève de seconde).

Le travail, qui recouvre alors cette activité autonome et régulière des élèves réalisée dans le but de répondre aux exigences des enseignants, vient renseigner les liens entre cette institution d'excellence et ceux qui la fréquentent. Malgré une mise sous pression ressentie les élèves font valoir "la bonne ambiance » qui existe au lycée en l'associant à la convivialité évoquée précédemment, comme à l'entraide et à la solidarité persistants entre les élèves. En même temps que l'établissement (à travers le proviseur et les enseignants) instaure une dynamique de travail exigeante (on doit consacrer du temps aux études, aux révisions avec méthode, à la gestion et à l'organisation de son emploi du temps), les élèves développent des stratégies en mettant en place une dynamique de travail collective. Ils se soutiennent pour progresser et faire face aux exigences de l'institution, et probablement aussi à celles de leurs parents au point de ne pas se sentir en compétition les uns avec les 


\section{eJRIEPS 47 Juillet 2020}

autres. Aucun élève de notre échantillon ne fait allusion à un climat de compétition. Si le fait de taire la compétition entre élèves peut tout aussi bien signifier qu'elle est neutralisée car incorporée, il semblerait que ce ne soit pas la concurrence qui caractérise l'excellence ici, mais un esprit d'entraide et de solidarité entretenu par les élèves, c'est à dire l'illustration que les avantages se cumulent à Henri IV.

\subsection{Une élite lycéenne.}

Même si «les établissements performants sont plus souvent, en moyenne, ceux qui accueillent un public de milieu aisé » (Duru-Bellat, 2003a, p. 580) nous pouvons nous demander qui se trouve dans ce lycée. On se pose cette question avant même d'y pénétrer. II est vrai qu'à quelques pas se situent la mairie du 5e arrondissement et plus bas la rue Descartes, le Ministère de l'Enseignement supérieur et de la Recherche, le Panthéon, la bibliothèque Sainte-Geneviève, les boutiques de luxe, des maisons d'édition, des librairies spécialisées, des compagnies bancaires et d'assurances, les rues Soufflot et Saint Jacques qu'arpentent les touristes en visite dans ce «beau quartier ». Le lycée Henri IV ne ressemble pas au commun des lycées. Cette impression se renforce au contact de ce qui l'environne, comme ces badauds qui semblent marcher vers le Jardin du Luxembourg avec l'hexis corporel propre aux catégories sociales les plus favorisées (Pinçon-Charlot \& Pinçon, 2007). Qu'en est-il des élèves ?

Premièrement les lycéens semblent avant tout parisiens (dans plus de $80 \%$ des cas). Ils proviennent de tous les arrondissements et plus précisément du $5 e$ (environ 20\%), du 13e et du $14 \mathrm{e}$ (plus de $7 \%$ pour chacun) puis des 1e, 20e 10e, 16e, 6e et 12e à parts relativement égales (de 5\% à 3\%). Pour le reste les élèves d'Henri IV sont issus d'abord en nombre de la Seine Saint Denis, des Hauts de Seine, du Val de Marne (une trentaine d'élèves dans chaque cas) et dans une moindre mesure de l'Essonne, du Val d'Oise (10 dans chaque cas) puis de la Seine et Marne, des Yvelines et même du Loir et Cher (de 6 à 1). Si on se rapporte aux travaux spécialisés qui font des arrondissements de Paris l'illustration d'un milieu social et plus globalement d'une symbolique sociale (Pinçon \& Pinçon-Charlot, 2014), on peut dire que la moitié des lycéens d'Henri IV appartient à des milieux aisés. Ceci s'obtient en considérant quelques indicateurs élaborés par l'INSEE et l'Atelier Parisien d'Urbanisme (revenu net moyen par foyer, prix moyen de l'immobilier, imposition annuelle, diplôme de l'enseignement supérieur). On peut par exemple présenter le $5 \mathrm{e}$ arrondissement (correspondant au lieu d'implantation du lycée et au premier foyer d'inscrits) comme l'une des zones les plus aisées de la capitale en raison d'une stratification sociale surreprésentant 


\section{eJRIEPS 47 Juillet 2020}

les cadres et professions intellectuelles supérieures (plus de 35\%). On distinguera au total deux grandes catégories au regard des indicateurs: d'un côté on retrouve des zones résidentielles favorisées qui participeraient à l'entretien d'un entre soi (les Hauts-de-Seine et les arrondissements 1, 3, 4, 5, 6, 7, 8, 9, 15, 16, 17 aux revenus nets moyens par foyer allant de 38000 euros à 82000 euros pour ne reprendre que cette variable), et de l'autre des aires en moyenne moins privilégiées. Si ce découpage renvoie à ce qui distingue classiquement l'ouest parisien, quelques prudences interprétatives s'imposent. On sait par exemple combien les arrondissements périphériques (deuxième groupe) contiennent des quartiers résidentiels, et à quel point les moyennes cachent des disparités (ce qui vaut également pour les banlieues du nord, du sud et de l'est). Ainsi, considérer que la moitié des lycéens appartient à des milieux aisés ne signifie pas que l'autre moitié est issue de familles défavorisées ; on notera cependant qu'un faible nombre de boursiers déclarés (7\% de notre échantillon). Si « habiter un beau quartier ou un quartier populaire, [...] vous marque déjà socialement » (Pinçon \& Pinçon-Charlot, 2008, p. 10), le lieu de résidence des élèves est donc un premier indicateur de leur origine sociale. Et cette première caractérisation s'accorde avec ce que l'on a collecté à propos des catégories socioprofessionnelles (CSP) occupées par les pères et mères des lycéens, indépendamment des moyennes relevées dans les zones de résidence. La composition sociale d'Henri IV reste spectaculairement au-dessus de ce que l'on constate dans la moyenne des lycées où plus d'un élève sur trois est enfant d'ouvriers, de retraités ou d'inactifs et où un élève sur quatre est issu d'un milieu de professions libérales ou de cadres (données RERS, 2014). A Henri IV plus de $70 \%$ des chefs de familles provient de cette catégorie (dans le cas du père et plus de $60 \%$ dans le cas de la mère). Cela implique que son excellence est potentiellement le produit d'une efficacité scolaire apportée par les élèves (Duru-Bellat, 2002b), desquels découlent quelques-uns des ingrédients de cette même excellence donc (vaste accès à la culture légitime, maitrise du métier d'élève, grandes facilités de compréhension, etc.). Le dépouillement des indicateurs relevant le niveau des activités extra scolaires confirme cet aspect. Avec presque $60 \%$ de lycéens répondant suivre au moins une activité (le minimum est souvent de deux) en dehors du cadre scolaire (associations et clubs, conservatoires, etc.), Henri IV présente la marque d'une pédagogisation des loisirs propice à la réussite (Daverne \& Dutercq, 2009). Ainsi ces lycéens sont en majorité inscrits dans un conservatoire de musique (où se pratiquent l'orchestre, le solfège, un instrument) puis de danse, ou au sein d'une structure dédiée: on allie ici pratiques sportives et pratiques artistiques, sans doute en raison d'une socialisation familiale appuyant les investissements 


\section{eJRIEPS 47 Juillet 2020}

de cette nature si l'on en juge par l'ancienneté des activités. La danse au sein d'une école ou d'un conservatoire (il s'agit le plus souvent de la pratique de la danse classique et/ou de la danse moderne et/ou du moderne jazz) s'impose comme l'activité extra-scolaire majeure dans notre échantillon $(9,7 \%)$; puis vient la pratique du tennis au second rang $(8,2 \%)$, devant la natation $(7 \%)$, le basket-ball $(4,4 \%)$, l'équitation $(3 \%)$, le football et le badminton (environ 2,5\%). C'est bien la diversité et l'originalité des activités sportives pratiquées qui caractérisent notre échantillon. On recense par exemple des pratiquants d'escrime, d'escalade, de rugby, de judo, de gymnastique, de tir à l'arc, de yoga, de boxe anglaise, de kung-fu, de cirque, de patinage artistique, de jujitsu. En couplant cette dimension à celle d'une pluriactivité, on confirme l'omnivorité comme caractéristique structurante des cultures sportives de milieux favorisés. En écho aux pratiques corporelles nous nous sommes intéressés au corps des élèves et à leur apparence physique, cette "façade personnelle, ensemble de signes incluant l'âge, le sexe, le vêtement, la taille, les comportements gestuels etc. » (Détrez, 2002, p. 128). Les observations du corps, un signe de statut (Boltanski, 1971, p. 232), livrent des informations à propos de conditions sociales d'existence des lycéens. Concernant la manière de se vêtir, nous n'avons jamais pu distinguer la marque des vêtements portés comme si elle ne faisait pas partie des stratégies de reconnaissance et de distinction, au contraire de ce qui se passe chez les lycéens étudiés par Pasquier (2005). Aucun élève ne portait non plus de survêtement avec la marque en évidence. Véronique, enseignante de SVT, raconte à ce sujet une anecdote : " J'ai un ancien élève qui venait de banlieue un peu difficile et qui me disait le code vestimentaire est pas du tout le même ici. II me disait, moi dans ma banlieue, la fierté c'était de porter des vêtements de marque. D'avoir les dernières Nike... et il disait, les gens ici ne sont pas du tout tape à l'œil dans leur façon de s'habiller. Ce n'est pas une priorité. Ils s'habillent correctement, ils font attention dans la plupart des cas mais il n'y a pas du tout de côté " je recherche l'apparence la plus visible".

Enfin, la majorité des élèves sont grands, minces, filiformes, élancés. Les enseignants d'EPS évoquent d'ailleurs la "stature " des élèves en entretien. L'un d'entre eux nous confiait à ce sujet : "Ce qui est flagrant c'est la stature des élèves, la plupart sont grands, minces, un peu musclés, développés, ici on a très peu d'élèves obèses... », et d'ajouter : “ J'ai l'impression qu'on a déjà une caractérisation des classes sociales » en expliquant qu'il serait capable d'identifier en début d'année, des élèves boursiers, qui viendraient des milieux plus défavorisés. Enfin, c'est surtout dans ce qui fait scolairement ces lycéens une élite que s'apprécie l'excellence d'Henri IV. Nos résultats montrent que quasi 100\% des 


\section{eJRIEPS 47 Juillet 2020}

élèves déclarent «naturellement » - adverbe signalé par les élèves eux-mêmes lors des passations directes de questionnaires - viser le baccalauréat en le décrochant avec une mention ( $90 \%$ visant une mention très bien). II ne fait pas de doute pour ces lycéens que l'objectif sera atteint, et qu'il n'y a apparemment pas de disjonction entre le travail fourni et les résultats obtenus contrairement à ce qui caractérise les élèves des milieux populaires (Barrère, 2008). L'ambition de ces lycéens se retrouve aussi lorsqu'on les sollicite à propos du temps post-baccalauréat : près de $60 \%$ des interrogés déclarent ainsi vouloir intégrer une Classe Préparatoire aux Grandes Ecoles (CPGE) du lycée. Sans constituer un groupe homogène qui ferait d'eux des éléments de la classe dominante, les adolescents d'Henri IV partagent différents types de ressources comme on l'a vu. C'est une population «à géométrie variable» (Heinich, 2004, p. 321) tant elle associe plusieurs principes d'excellence : le prestige social, le prestige culturel transmis par la famille mais que les élèves s'approprient dans leurs loisirs ou leurs pratiques extra-scolaires. Les élèves du lycée Henri IV ont aussi cette caractéristique particulière conférée aux élites, celle de se savoir en faire partie. A l'image de Bastien, élève de terminale rencontré à l'occasion du cours de rock en Association Sportive, les élèves savent qu'ils ne sont pas là par hasard. En cela, appartenir à l'élite revient bien à être «tenu pour tel à la fois par ceux qui en font partie (cooptation et par ceux qui n'en font pas partie ») (Meuret, 2011, p. 41). En reconnaissant que sa trajectoire scolaire est déjà toute tracée, organisée et calculée lorsqu'il nous confie « avec mes parents on savait déjà que j'irai ici. Ce n'est pas un hasard d'être là », Bastien a en lui ce sentiment d'appartenir à l'élite, ce sentiment « d'auto-perception » dont rend compte Elias (1985). L'entrée en seconde se fait en effet sur dossier scolaire comme c'est le cas au lycée Louis Legrand. Ce sont les deux établissements de Paris qui ont le droit de recruter leurs élèves de cette manière. Véronique, enseignante de SVT considère qu'il s'agit là d'un « recrutement très particulier » dans la mesure où « ça touche un peu un fonctionnement du Privé sauf que c'est gratuit ». Nous n'avons pas pu assister aux sélections des candidatures qui se font sur les appréciations des bulletins scolaires et les notes obtenues au collège. Mais lors d'un entretien formel, le proviseur du lycée nous confiait que l'on entre à Henri IV « parce qu'on est très bon, que l'on a un projet ambitieux et des exigences par rapport à soi-même " et que ce qui compte c'est l'attitude, le comportement plus que les notes. Si nous n'avons pas pu analyser ce travail de recrutement, il semble que quelque chose relève, comme pour les classes préparatoires, de la « recherche de dispositions adéquates » (Darmon, 2013, p. 31). Cela fait de cette élite lycéenne un groupe d'excellence constitué antérieurement aux filières d'excellence que sont 


\section{eJRIEPS 47 Juillet 2020}

les CPGE par exemple qui combine logique stratégique et logique de subjectivation. En 2014 , c'est en effet $52,17 \%$ des collégiens passant en seconde qui ont été admis au lycée Henri IV (chiffres tirés du site internet du lycée). Et si certains élèves font leur entrée en seconde en provenant des « cordées de la réussite », c'est-à-dire d'établissements moins favorisés, très vite ils vont se fondre dans la masse, intégrer les codes et les attentes de l'institution. Le sociologue américain Wilson (1959) insiste dans ses travaux consacrés aux projets de jeunes lycéens, sur « la pression normative du groupe d'appartenance et l'impact de ses valeurs sur ses membres » (Duru-Bellat, 2003b, p. 184). Le school mix va jouer sur le niveau d'aspiration général des élèves en même temps que sur leur comportement. Marie Duru-Bellat (2003a) rappelle que «à niveau scolaire comparable, un jeune de milieu populaire scolarisé dans un collège " chic » fait montre d'un niveau d'aspiration scolaire plus élevé (se rapprochant de ses pairs de milieu plus favorisé) que s'il était dans un établissement plus populaire » (p. 580). Il va se produire ainsi une sorte de mimétisme quant aux manières d'être et de travailler, au langage aussi. Valérie, enseignante d'EPS dans l'établissement, déclare : «[...] on a déjà une caractérisation des classes sociales ; et pour les élèves qui viennent de milieux plus défavorisés ; qui ont été recrutés sur dossier, il y a un mimétisme qui se fait et on retrouve un comportement qui, voilà, qui est celui qu'on trouve ici au lycée, j'ai l'impression ». Sylvie, enseignante de SVT explique aussi : «Parfois on en repère quelques-uns qui viennent de la cordée, c'est-à-dire qu'ils sont rentrés en seconde en venant d'établissements moins favorisés ; mais j'avoue qu'en première généralement ils sont intégrés, c'est rentré dans l'ordre. Après ça, j'en ai un ou deux que j'ai aidé pour le voyage. Généralement je vois ces différences au moment du voyage, mais pour le reste... je le vois parfois au niveau de langue...hein...l'écrit, il y en quelques-uns pour lesquels le français n'est pas la langue parlée à la maison ». II faudrait en passer par une étude qualitative du métier de lycéen à Henri IV pour proposer une analyse consistante de ce qui s'y joue. Mais si on croise nos récoltes de terrain avec des résultats d'études plus poussées (comme Dutercq \& Daverne, 2009), on voit bien que ces lycéens concentrent ce qui fait l'élite lycéenne soit l'autonomie relative, la solidité du projet, la volonté de réussir mais dans le plaisir. Une telle précocité pourrait bien être la marque d'une distinction. Comment se décline-t-elle en EPS ? Que vont faire les enseignants d'une EPS qui se situe à l'interface de dispositions incorporées par les élèves (lors de leurs socialisations primaire et secondaire) ?

\subsection{L'EPS et l'élite lycéenne d'Henri IV}




\section{eJRIEPS 47 Juillet 2020}

\subsubsection{L’équipe enseignante}

Lors de l'année scolaire 2014-2015, l'équipe EPS du lycée Henri IV se composait de onze membres : six hommes (dont un professeur stagiaire) et cinq femmes. Au fur et à mesure de l'enquête, l'équipe a connu quelques remaniements (renouvellement du professeurstagiaire, nouvelles nominations suite à deux départs en retraite, nomination d'un Titulaire de Zone de Remplacement à l'année...). Les tableaux I et II dressent les caractéristiques des enseignants d'EPS de l'établissement au moment de l'enquête. Nous distinguons les enseignants que nous avons observés en les indiquant en gras.

Tableau I. Caractéristiques des enseignants d’EPS du lycée Henri IV (2014-2015).

\begin{tabular}{|c|c|c|c|c|c|c|c|c|c|c|c|}
\hline \multicolumn{12}{|c|}{$\begin{array}{l}\text { Date de naissance et date d'entrée dans l'établissement } \\
\text { des enseignants de l'équipe EPS (2014-2015) }\end{array}$} \\
\hline & Monique & Luc & Valérie & Françoise & Jocelyne & Annick & Claude & Éric & Joris & Thibault & Adrien \\
\hline Naiss & 1955 & 1954 & 1961 & 1959 & 1958 & 1976 & $?$ & $?$ & 1964 & $?$ & 1985 \\
\hline $\begin{array}{c}\text { Entrée } \\
\text { établ }\end{array}$ & 1984 & 1983 & 2007 & 2005 & 2000 & 2008 & $?$ & $?$ & 2005 & 2011 & 2014 \\
\hline
\end{tabular}

Tableau II. Caractéristiques des enseignants d'EPS du lycée Henri IV (2014-2015).

\begin{tabular}{|c|c|c|c|c|c|c|c|c|c|c|c|}
\hline \multicolumn{12}{|c|}{$\begin{array}{c}\text { Structure de formation, concours, diplômes obtenus, statut. } \\
\text { des enseignants de l'équipe EPS (2014-2015) }\end{array}$} \\
\hline & Monique & Luc & Valérie & Françoise & Jocelyne & Annick & Claude & Éric & Joris & Thibault & Adrien \\
\hline Formation & CREPS & CREPS & CREPS & STAPS & UREPS & STAPS & UREPS & UREPS & STAPS & STAPS & STAPS \\
\hline Concours & $\begin{array}{c}\text { Concours } \\
\text { national } \\
\text { prof. } \\
\text { adjoint }\end{array}$ & $\begin{array}{c}\text { Capeps } \\
\text { ext }\end{array}$ & $\begin{array}{c}\text { Capeps } \\
\text { ext } \\
\text { AG int }\end{array}$ & $\begin{array}{c}\text { Capeps } \\
\text { ext }\end{array}$ & $\begin{array}{c}\text { Capeps } \\
\text { ext }\end{array}$ & $\begin{array}{c}\text { Capeps } \\
\text { ext } \\
\text { AG int }\end{array}$ & $\begin{array}{c}\text { Capepx } \\
\text { ext }\end{array}$ & $\begin{array}{c}\text { Capeps } \\
\text { ext }\end{array}$ & $\begin{array}{c}\text { Capeps } \\
\text { ext } \\
\text { AG int }\end{array}$ & $\begin{array}{c}\text { Capeps } \\
\text { ext }\end{array}$ & \\
\hline Statut & $\begin{array}{c}\text { CE EPS } \\
\text { Exc }\end{array}$ & $\begin{array}{c}\text { PrEPS- } \\
\text { Hcl }\end{array}$ & AG hel & PrEPS-hcl & $\begin{array}{c}\text { PrEPS- } \\
\text { hcl }\end{array}$ & $\mathrm{AG}$ & $\begin{array}{c}\text { PrEPS- } \\
\text { hcl }\end{array}$ & $\begin{array}{c}\text { PrEPS- } \\
\text { hcl }\end{array}$ & $\mathrm{AG}$ & $\begin{array}{c}\text { PrEPS } \\
\text { TZR }\end{array}$ & Stg \\
\hline
\end{tabular}

Avec sept enseignants (quatre femmes et trois hommes) ayant atteint la hors-classe, Monique étant professeure Chargée d'Enseignement (CE) EPS, on peut dire, au contraire des établissements des Zones d'Education Prioritaire (ZEP) dans lesquels se concentrent les enseignants les moins expérimentés et les plus jeunes (Kherroubi \& Rochex, 2004), que ces indicateurs rendent compte de la stabilité et de l'expérience professionnelle de l'équipe pédagogique. C'est un groupe qui se caractérise par son ancienneté professionnelle. 


\section{eJRIEPS 47 Juillet 2020}

$\mathrm{Si}$ on considère que certaines activités physiques induisent des transformations motrices particulières ainsi qu'un type de rapport au corps que l'on a choisi de valoriser, des significations sociales pouvant être associées à ces activités (Combaz, 2008, p. 72), à quel type de rapport au corps les pratiques sélectionnées et organisées dans le cadre des leçons d'EPS renvoient-elles? Questionner le contenu de la programmation EPS de l'établissement, c'est interroger la culture scolaire qui a choisi d'être enseignée dans la mesure où les activités physiques portent chacune des caractéristiques intrinsèques spécifiques et renvoient ce faisant, à des " modes de vie et des valeurs pouvant être culturellement très distinctifs » (Combaz \& Hoibian, 2009, p. 190). Etablie en tenant compte des injonctions programmatiques et du cadre institutionnel de l'EPS au lycée (B.O des 29 avril $2009 n^{\circ} 4$ et du 19 février $2010 n^{\circ} 2$ ), la programmation tient d'abord compte des installations disponibles que les enseignants jugent positivement. Lors de l'année 20142015, la programmation des activités (APSA) reposait sur les activités suivantes: athlétisme, natation sportive et natation de durée, escalade, acrosport, danse, tennis de table, volley-ball, handball, musculation et step. A dessein de comparaison, on peut s'appuyer sur l'enquête dirigée par Benhaïm-Grosse (2007) effectuée pour le Ministère de l'Education Nationale et qui donnent un aperçu de ce qui est majoritairement programmé en EPS dans les établissements du secondaire (collèges et lycées confondus). On peut ainsi considérer qu'il s'agit là d'une offre classique dans la mesure où cette offre correspond aux activités pratiquées dans plus de $80 \%$ des établissements du second degré (BenhaimGrosse, 2007): "Elles nécessitent des compétences détenues par presque tous les enseignants et des infrastructures qui existent dans presque tous les établissements : athlétisme, badminton, volley-ball, basket-ball, handball ou tennis de table » (BenhaïmGrosse, 2007, p. 75). De même, si la musculation fait partie des APSA dites " discriminantes » et que l'on retrouve cette activité dans le cadre de la programmation du lycée Henri IV, cette activité est malgré tout « très fréquemment programmée en LP et en lycées » (Benhaïm-Grosse, 2007, p. 75). Les choix qui ont été faits par les enseignants au sujet de la programmation des APSA oscillent entre une logique normative (la programmation respecte les injonctions programmatiques et en cela le curriculum formel prescrit officiellement, puisqu'elle s'appuie sur la liste nationale des APSA et sur le référentiel de compétences attendues) et une logique matérielle. Jamais ils ne font allusion aux caractéristiques sociales du public ou ne se réfèrent à un type de rapport au corps à valoriser. Valérie explique par exemple : "On reste toujours dans le cadre des textes et dans un cadre confortable qui est celui d'utiliser nos installations et pas celles de 


\section{eJRIEPS 47 Juillet 2020}

l'extérieur » et Françoise, sa collègue, de confier : «Après il n'y a pas forcément d'originalité par rapport à d'autres établissements puisqu'on se base sur la programmation EPS, on ne peut pas en sortir ». II semble aussi qu'une certaine habitude persiste, que le confort des installations de l'établissement limite originalité et innovations. Mais l'équipe EPS fait également valoir la compétence des enseignants, ou leur «spécialité » comme un déterminant de la programmation : « Je trouve que par la force des choses, on tourne un peu en rond mais bon. Et c'est comme ça. C'est dû aux installations matérielles. Il y a eu du judo ici avant, mais très peu, ça dépend aussi de la spécialité des professeurs » (Monique). On pourrait alors penser, au vu de l'ancienneté de l'équipe, que le traditionalisme est importé par les enseignants eux-mêmes.

Enfin, les représentations du sport fédéral qui semblent servir de division dominante de l'espace. La plupart des activités programmées (athlétisme, tennis de table, volley-ball, handball, natation) s'alignent sur les normes de l'institution sportive (Parlebas, 1986 ; 1960). Cependant, l'affichage de ces APSA ne préjuge en rien des contenus d'enseignement réels. Si la «mise en forme stylistique des APS» (Marsault, 2005, p. 11) ne révèle pas de traitement social de l'activité particulier et ne permet pas de saisir l'intérêt éducatif visé par les enseignants, on sait que les APSA peuvent être transformées, détournées de leurs buts initiaux selon les normes culturelles des pratiquants (Poggi, 2002, p. 55). A quoi ressemblent alors les leçons d'EPS ? Comment se déroulent-elles ?

\subsection{Les modes organisationnels des leçons : mesure, cadence et vitesse d'apprentissage}

2.4.1. Cadence, mesure et contrôle des corps

Les leçons d'EPS du lycée Henri IV renvoient à la typologie structurelle observée dans les établissements ECLAIR étudiés par Vors (2013) : prise en main des élèves, cours dialogué et retours au calme. L'observation des leçons de Monique, Valérie et Luc durant un cycle d'activité, comportent toutes une «trame». Une structure répétée d'une leçon à l'autre au cours du cycle. Les enseignants prennent en main les élèves, annoncent les thèmes de la leçon, lancent l'échauffement puis les situations d'apprentissage pour terminer la leçon par un bilan collectif. Par exemple, toutes les leçons de Monique en tennis de table se composent d'une prise en main, d'un échauffement, de situations d'apprentissage et d'un retour au calme avec bilan collectif. Chacune des leçons se structure autour de trois types de tâches : 


\section{eJRIEPS 47 Juillet 2020}

- des tâches individuelles lors de l'échauffement où il s'agit de se mettre en activité. L'objectif est alors de porter son attention sur le travail des appuis.

- des tâches de coopération au cours desquelles les élèves exécutent des gestes techniques (coup droit, revers, service) en s'adaptant à leur partenaire pour favoriser la répétition du geste. L'objectif est régulièrement de réaliser le plus grand nombre d'échanges.

- des matchs sous formes de montante/descendante.

Au cours du cycle de tennis de table, Luc propose deux types de tâches :

- des situations de jeu en coopération : les élèves sont parfois tuteurs et font répéter un geste technique à leur camarade au cours de situations de coaching; ils sont parfois partenaires sur des matchs en double.

- des situations de matchs : au cours desquelles les élèves sont davantage dans une attitude compétition en raison de la logique de l'activité. Sur des matchs organisés en poule ou bien des montantes/descendante, les élèves s'efforcent de rechercher le gain du match pour ne pas descendre dans le classement. Nos observations montrent qu'à aucun moment dans les leçons, il n'y a de conflit à gérer, d'indiscipline, de décrochage, de manquement à la règle ou à l'autorité. C'est une «EPS où on ne crie pas ". Bien sûr, quelques bavardages peuvent se faire entendre mais de manière assez brève et discrète. Les enseignants s'arrêtent alors pour reprendre ces bavardages et faire remarquer aux élèves " qu'ils sont bien énervés aujourd'hui ". On peut penser aussi que les élèves mettent en place "un réseau buissonnier de communication" (Depoilly, 2014) qui aurait la spécificité de ne jamais ou très rarement perturber l'activité enseignante. Mais ce qui était bien visible c'était le calme, l'ambiance studieuse de la classe en même temps que le rythme de la leçon. Calme et rythme. Mesure et cadence. Les élèves se mettent rapidement au travail, passent d'une situation d'apprentissage à une autre, en même temps qu'ils écoutent avec attention les consignes, maîtrisent leurs émotions pour satisfaire les exigences et les attentes de l'enseignant. Ici, les élèves n'explosent pas de joie; ils ne sont pas dans l'ostentation de leurs émotions. Les corps sont des corps maîtrisés. Pas autant que le sont les corps des danseurs qu'a étudiés Isabelle Queval (2008), mais on voit là des corps dans la rectitude, dans « le beau port de tête » comme le décrit Jocelyne. Jamais ils ne s'avachissent. Jamais ils ne s'affalent lors des phases de regroupement, jamais ils ne s'allongent de tout leur long au sol pour écouter les consignes. En dehors des situations ils restent dans une attitude de respect qui passe par la posture du corps: ils sont disciplinés dans leurs attitudes, disciplinés dans leur corps. Le rythme de la leçon tient en premier lieu aux interactions que 


\section{eJRIEPS 47 Juillet 2020}

l'enseignant entretient avec les élèves. Ce rythme imprimé à la leçon sert, non pas à engager les élèves dans l'activité puisqu'ils s'y impliquent. Mais ce tempo sert d'une part, à maintenir les élèves en activité et dans les tâches d'apprentissage, et d'autre part, à soumettre les corps aux exigences des tâches. Luc, Monique et Valérie ponctuent la leçon d'interventions verbales qui encouragent les élèves dans les tâches motrices. Ce sont des communications d'animation (Marsenach, 1989) qui motivent les élèves et soutiennent leur implication: «leur impact est plus affectif qu'informatif et leur fonction motivationnelle » (Marsenach, 1989, p. 9). Si de rares rappels à l'ordre ou « communication d'autorité » surviennent, ils soulignent le temps qui est perdu sur la leçon. Ces rappels sont distillés au moment des consignes, juste avant la tâche, souvent d'ailleurs en tout début de leçon. Le ton est alors ferme au point qu'il impose un silence. Les enseignants ne crient jamais. Ils haussent le ton ou marquent une pause en fixant l'élève à qui ils destinent la remarque. Or, c'est bien la structure des leçons (à travers leur cadre) mais aussi les injonctions des enseignants qui renforcent l'assujettissement des corps des élèves. Gasparini et Pardo (2000) montrent d'ailleurs comment, dans les cours d'EPS des collèges en banlieue lyonnaise, «les séances structurées dans leurs temporalités, demandent un assujettissement des corps aux injonctions du professeur et un respect strict des règles qui vont dans le sens d'un renforcement du mode scolaire de socialisation, ce qui ne tend pas à aider les élèves (notamment ceux qui ont des dispositions issues de modes de socialisation éloignés de la logique scolaire) à mieux se "comporter" " (Faure \& Garcia, 2003, p. 89). La mesure couplée à la cadence de la leçon permet de faire progresser la leçon rapidement en faisant évoluer les situations, en centrant les contenus d'enseignement sur la progression motrice. Mais bien que le lycée Henri IV se distingue des établissements classés en ZEP en raison de l'homogénéité sociale de son public, ses leçons d'EPS se rapprochent des contextes difficiles à cause de l'hétérogénéité motrice des élèves. Si près de $60 \%$ des élèves ont déclaré avoir une pratique physique extérieure, les enseignants restent néanmoins confrontés à des élèves ayant des difficultés motrices: les comportements varient au plan moteur. On peut voir des corps rigides lors des leçons bien que cela dépende des activités, en particulier en danse et en acrosport. Pour certains lycéens, les mouvements semblent retenus, étriqués et quelques lycéens (ce n'est pas la majorité) restent peu à l'aise avec leur corps. Pourtant l'hétérogénéité motrice n'empêche pas les leçons « d'aller vite » au moyen de la multiplication des variables didactiques dans les situations, de temps de rassemblements collectifs et de temps de questionnement. A chacune des leçons, lorsque les élèves sont en situations, Luc reste en retrait du groupe- 


\section{eJRIEPS 47 Juillet 2020}

classe et observe ce qui se déroule sur les tables depuis le bureau. Pour réguler l'activité des élèves, il entrecoupe les séquences de matchs, de rassemblements collectifs.

\subsubsection{Le temps et la vitesse d'apprentissage}

Cette autodiscipline rend possible l'établissement d'un apprentissage réfléchi et autonome en raison des capacités cognitives des élèves, du vocabulaire qu'ils maîtrisent et de leur pratique physique extérieure qui leur a sans doute permis de s'approprier des méthodes de travail, la rigueur et la persévérance. Valérie évoque une « capacité de rebond " qui l'a frappée lorsqu'elle est arrivée dans l'établissement : « II y a une capacité d'attention et une capacité de rebond qui est quand même assez extraordinaire [...] l'apprentissage se faisait de façon plus rapide ». Et si les situations évoluent rapidement, les élèves prennent le temps de comprendre, de répéter et d'apprendre. Ils ont bien saisi que leur progrès, voire leur performance en EPS, passent par une accumulation du temps dans la pratique : ils consentent par exemple à répéter un geste technique en tennis de table ou un élément gymnique en acrosport. Par ailleurs, les modalités d'apprentissage en EPS semblent être identiques aux autres disciplines. Véronique, enseignante de SVT nous explique que la vitesse d'apprentissage et la compréhension des élèves sont des exigences communes à l'équipe éducative de sa discipline : «On exige d'eux de travailler surtout rapidement. Nous en SVT on donne souvent beaucoup d'exercices dans un temps limité. Ils doivent apprendre à travailler vite et précisément. On est très exigeant sur le vocabulaire utilisé, sur la démarche. Quand ils ont appris il faut qu'ils soient capables de nous montrer qu'ils ont compris et respecté les consignes ». La vitesse d'apprentissage et la vitesse d'exécution sont des traits caractéristiques des élèves du lycée dans leur ensemble puisque de la même manière, Françoise explique : «Ils savent travailler vite, faut que ça avance. Ils ont du mal un peu parfois à se canaliser pour dire bon on attend un peu ceux qui sont en difficulté ». Laura me confie également: «Ils calculent très vite, ils comprennent très vite et après ils entrent en action rapidement. Donc il y a plus de temps de pratique et donc il y a plus de chances de progresser ». En leçon 2, après l'échauffement, Valérie annonce que le thème de travail est le maintien de l'appui tendu renversé (ATR). Elle définit les enjeux d'apprentissage du dispositif. L'objectif de la situation est de maintenir trois secondes un ATR. Pour mettre en place la situation de travail, Valérie questionne d'abord l'ensemble de la classe : «Comment on doit monter et revenir ? ». Elle valide ou invalide les réponses et insiste sur des contenus d'enseignement moteurs : «On monte en fouettant la jambe, le corps aligné, les mains aux oreilles, les bras tendus, on verrouille les coudes et les épaules, 


\section{eJRIEPS 47 Juillet 2020}

on a les mains dans l'axe, les jambes sont serrées trois secondes ». Valérie fait démontrer la situation par une élève qui réalise l'ATR. Elle montre où il faut se placer pour parer le partenaire : «Sur l'ATR, le pareur est devant, on maintient trois secondes même si elle [en parlant de l'élève qui réalise l'ATR] sait tenir la position». Les élèves ont pour consigne d'effectuer un ATR avec un pareur. Mais ils peuvent aussi s'exercer contre un tapis dima disposé contre des espaliers. Les élèves essaient ensuite par deux de réaliser un ATR et de maintenir la position trois secondes. Valérie passe dans quelques groupes pour corriger des positions, redonner des contenus moteurs. On voit alors des élèves qui rencontrent quelques difficultés : certains appréhendent la bascule avant du bassin, certains basculent, montent à la verticale mais écartent les jambes et s'écroulent en raison d'un manque de tonicité (les coudes ne sont pas verrouillés, le corps n'est pas gainé). D'autres encore ne maîtrisent pas le retour et reviennent les deux jambes en même temps. Mais de manière générale, ils démarrent tous en fente avant, les bras collés aux oreilles et se concentrent avant l'exécution. Tous prennent le temps de répéter l'ATR quand ils ne réussissent pas la bascule du bassin et qu'ils s'écroulent en mettant en rapport les contenus d'enseignement de l'enseignant et leur activité motrice. Ils décident seuls d'aller retravailler le passage à l'ATR avec un aménagement matériel mis à disposition par Valérie pour revenir ensuite au travail avec pareur. L'enseignante ne manipule pas de dispositifs matériels à l'intérieur des tâches mais recourt à une activité médiatrice : elle échange avec les élèves, elle les questionne, elle fait verbaliser les solutions expérimentées, trouvées : «Qu'est-ce qui fait que tu as réussi là et que tu as tenu plus longtemps ? », « J'ai serré les jambes, je n'ai pas redressé la tête». Enfin, Valérie insiste sur des contenus moteurs lorsqu'elle passe dans les groupes, pour encourager, et réguler le comportement moteur des élèves et redonner des contenus sécuritaires: "Pense à verrouiller les coudes; on serre le ventre; on contracte les abdominaux; allez ! ; soyez vigilant, soyez vigilant à ce que vous faites et votre partenaire ». Les leçons d'EPS révèlent leur singularité lorsqu'on se réfère aux capacités et aux attitudes des élèves. Ces derniers prennent le temps de faire, de comprendre, de répéter et d'apprendre. C'est alors un rapport au temps qui semble être optimisé, maximisé. Si l'excellence scolaire se mesure dans le rapport au temps, l'excellence en EPS repose aussi sur la vitesse des apprentissages comme si la vitesse était « la forme ultime de l'excellence. Comprendre vite, faire vite » (Darmon, 2013, p. 153).

\section{Discussion et conclusion}




\section{eJRIEPS 47 Juillet 2020}

Nous avons essayé dans cet article de rendre compte d'un objet encore méconnu. Nous avons travaillé avec des techniques de recherche variées, utilisé des cadres théoriques permettant une forme d'interprétation contrôlée tout en adoptant une démarche fondée sur une vision ascendante des enquêtes. Cette posture a conduit à la réalisation de passations de questionnaires, d'observations outillées qu'elles soient directes ou indirectes. Et si cette plongée n'est pas restituée ici dans son intégralité, elle permet un premier portrait de l'EPS qui s'enseigne dans un contexte d'excellence où les très bons élèves sont nombreux. Les données recueillies donnent une vision plus précise de ce qu'est un « très bon élève en EPS »: il se caractérise par son ambition scolaire, sa capacité de répétition, de compréhension et d'adaptation, par sa capacité de travail en autonomie et en collaboration avec d'autres élèves. Nous avons mis à jour une EPS fortement investie, respectée comme le sont ceux qui l'enseignent et le rapport au temps y est maximisé au profit des apprentissages. Les enseignants d'EPS structurent et cadencent les leçons en faisant face à une hétérogénéité de comportements moteurs ; et les normes de l'excellence en EPS reposent davantage sur les compétences et dispositions manifestes des élèves que sur la programmation d'activités typiques des classes dominantes ou innovantes. On pourrait bien s'imaginer que cette discipline ressemble aux autres : l'excellence n'est pas la sienne ni celle de l'enseignant, mais plutôt le produit de ce qu'imposent les élèves et leurs dispositions. Les enseignants ne sont pas pris dans l'agitation d'une classe qu'il s'agit de gérer et font autant preuve de flegme que d'autorité. Les comportements dociles des élèves, leur sérieux, leur investissement constant, ces comportements qui préexistent d'ailleurs à la leçon, font que celle-ci est « facile » à mettre en œuvre, « facile » à gérer, à « dérouler » ; ce sont des leçons « confortables » selon Valérie parce qu'il n'y a pas à être sur le « quivive », à l'affût du moindre débordement, de la moindre bagarre, du moindre mot de travers à reprendre, à corriger, à sanctionner. Ce confort dans l'enseignement n'est pas une spécificité des leçons d'EPS dans la mesure où Sylvie, enseignante de SVT déclare « qu"ici c'est beaucoup moins fatiguant ", " il n'y a pas d'imprévus ici ou peu d'imprévus. II y plus de travail de préparation, de corrections mais c'est beaucoup moins fatiguant. Même si on a quarante élèves et que j'en avais vingt ou vingt-cinq dans le XXème ».

A ce titre on voit bien, en EPS comme ailleurs, l'influence des milieux familiaux comme des dispositions qu'ils détiennent : nos enquêtes montrent en effet que ces très « bons élèves » d'Henri IV pratiquent des activités physiques et sportives en dehors de l'école, ce qui constitue sans doute en la matière une ressource utile en EPS (en raison d'une socialisation précoce aux activités, d'un développement moteur enclenché voire entretenu, etc.). Issus 


\section{eJRIEPS 47 Juillet 2020}

de milieux privilégiés composés d'intellectuels et de cadres mixtes, la plupart des élèves d'Henri IV ressembleraient de façon prévisible (Mennesson \& Julhe, 2012) à des détenteurs d'une culture somatique et/ou artistique qui paie en EPS. Même si notre étude ne permet pas de certifier ce poids parce que ces activités relèvent d'une persuasion douce dans les familles (Daverne \& Dutercq, 2009) la rendant difficile à pointer, il constitue sans doute une piste intéressante. En l'explorant et en la vérifiant, de prochains travaux montreraient que l'EPS ressemble à certains égards à la plupart des disciplines scolaires dont la réussite des apprentissages repose en partie sur une pédagogisation familiale d'activités extrascolaires qui consisterait à tirer profit des activités extrascolaires pour en faire des ressources scolaires, des «loisirs rentables » (Daverne et Dutercq, 2009, p. 26) dans le parcours scolaire des élèves.

\section{Bibliographie}

Amade-Escot, C. (dir.) (2003). Didactique de l'éducation Physique. Etat des recherches. Paris : Édition Revue EPS.

Amade-Escot, C., Verscheure, I., \& Devos, O. (2002). «Milieu didactique » et "régulations » comme outils d'analyse de l'activité du professeur en éducation physique. In: Les dossiers des sciences de l'éducation, (8). Didactique des disciplines scientifiques et technologiques : concepts et méthodes, 87-97.

Barrère, A. (2008). De l'héritier au forçat. Vacarme, 3(44), 24-28.

Barrère, A. (2002). Un nouvel âge du désordre scolaire : les enseignants face aux incidents. Déviance et Société, 26(1), 3-19.

Benhaïm-Grosse J. (dir.). (2007). Image du sport scolaire et pratiques d'enseignement au collège et au lycée. Les Dossiers. Enseignement scolaire, Ministère de l'Éducation nationale, Direction de l'évaluation, de la prospective et de la performance, 190.

Berthelot, J-M. (1983). Le piège solaire. Paris : Presses universitaires de France.

Boltanski, L. (1971). Les usages sociaux des corps. Annales. Economie, Sociétés, Civilisations, 1, 205-231. doi : https://doi.org/10.3406/ahess.1971.422470

Bonniot-Paquien N. (2009). Les enseignants d'EPS face aux élèves qui décrochent de l'activité : intervention selon le sexe des élèves et discours relatifs aux comportements observés. STAPS, 2(84), 77-92.

Bourdieu, P. (1997). Méditations pascaliennes. Paris : Le Seuil 


\section{eJRIEPS 47 Juillet 2020}

Bourdieu, P. (1989). La Noblesse d'État. Grandes écoles et esprit de corps. Paris : Les Éditions de Minuit.

Bressoux P. (1994). Les recherches sur les effets-école et les effets-maîtres. Revue française de pédagogie, 108, 91-37.

Cacouault-Bitaud, M., \& Oeuvrad, F. (2009). Sociologie de l'éducation. Paris: La Découverte.

Céfaï D. (2003b). L'enquête de terrain. Paris, France : La Découverte.

Chartier, M. (2016). Une « non-maîtresse dans les écoles. Ethnographie de la scolarisation des enfants du voyage ». Dans J-P. Payet (dir.), Ethnographie de l'école, les coulisses des institutions scolaires et socio-éducatives (p. 83-97). Rennes : Presses universitaires de Rennes

Chevallard, Y. (1999). L'analyse des pratiques enseignantes en théorie anthropologique du didactique. Recherche en didactique des mathématiques, 19(2), 221-266.

Cogérino G., \& Mnaffakh H. (2008). Evaluation, équité de la note en EPS et norme d'effort. Revue française de pédagogie, 164, 111-122.

Cohen J. (2006). Social, emotional, ethical and academic education: Creating a climate for learning, participation in democracy and well-being. Harvard Educational Review, 76(2), Summer, 201-237.

Combaz, G. (2008). La légitimité de la culture scolaire mise à l'épreuve : l'exemple de l'EPS en France. Dans J. Bréhon et N. Niedzwialowska (coord.), Enseigner l'EPS : entre le dire et le faire. (Dossier EPS, $\mathrm{n}^{\circ} 77$, p. 71-77). Paris, France : Éditions Revue EPS.

Combaz G., \& Hoibian O. (2009). La légitimité de la culture scolaire mise à l'épreuve. L'exemple de l'éducation physique et sportive dans le second degré en France. Education et sociétés, 1(23), 189-206.

Copans, J. (1966). La monographie en question. L'Homme, 6(3). 120-124.

Cousin O. (1993). L'effet établissement. Construction d'une problématique. Revue française de sociologie, 34(3), 395-419.

Darmon M. (2010). Des jeunesses singulières. Sociologie de l'ascétisme juvénile, Agora débats/jeunesses, 3(56), 49-62.

Darmon M. (2013). Classes préparatoires. La fabrique d'une jeunesse dominante. Paris: La Découverte.

Daverne C., \& Dutercq Y. (2009). Les élèves de l'élite scolaire : une autonomie sous contrôle familial, Cahiers de la recherche sur l'éducation et les savoirs, 8, 17-36. 


\section{eJRIEPS 47 Juillet 2020}

Depoilly, S. (2014). Les garçons et l'école : rapports sociaux de sexe et rapports de classe. Travail, genre et sociétés, 31(1), 151-155. doi:10.3917/tgs.031.0151.

Détrez, Ch. (2002). La construction sociale du corps. Paris : Le Seuil.

De Saint Martin M. (2009). Les jeunes favorisés et les autres (entretien), Agora, 3(53), 5971.

De Saint Martin M. (2005). Méritocratie ou cooptation ?, Revue internationale d'éducation de Sèvres, 39, 57-66.

Draelents H. (2010). Les effets d'attraction des grandes écoles. Excellence, prestige et rapport à l'institution, Sociologie, 1(3), 337-356.

Dubet F. (1991). Les lycéens. Paris : Seuil.

Dufrasne, CL. (1982). Centre national de la recherche scientifique. Centre de sociologie des organisations. Revue française de pédagogie, 61, 75-77.

Duru-Bellat, M. (2003a). Actualité et nouveaux développements de la question de la reproduction des inégalités sociales par l'école, L'orientation scolaire et professionnelle [en ligne], 32(4), 571-594.

Duru-Bellat, M. (2003b). Les apprentissages des élèves dans leur contexte : les effets de la composition de l'environnement scolaire. Carrefours de l'éducation, 16(2), 182-206. doi:10.3917/cdle.016.0182.

Duru-Bellat M. (2002a). Effets maîtres, effets établissements : quelle responsabilité pour l'école ?. Revue suisse des sciences de l'éducation, 23(1), 321-337.

Duru-Bellat, M. (2002b). Les inégalités sociales à l'école: Genèse et mythes. Paris : Presses universitaires de France.

Dutercq Y., \& Daverne C. (2009). Les parcours composites de l'élite lycéenne. Les Sciences de l'éducation - Pour l'Ere nouvelle, 42(4), 17-37.

Elias N. (1985). La Société de cour. Paris : Flammarion, coll. Champs, (1969).

Faure, S., \& Garcia M-C. (2003). Le corps dans l'enseignement scolaire : regard sociologique. Revue française de pédagogie, 144, 85-94.

Félix, C., Amigues, R., \& Espinassy, L. (2014). Observer le travail enseignant. Recherches en éducation. Recherches en Éducation, 19, 52-62.

Ferrand M., Imbert F. et Marry C. (1999). L'excellence scolaire : une affaire de famille. Le cas des normaliennes et normaliens scientifiques. Paris : L'Harmattan.

François, J., \& Poupeau, F. (2008). Les déterminants socio-spatiaux du placement scolaire: Essai de modélisation statistique appliquée aux collèges parisiens. Revue française de sociologie, 49(1), 93-126. doi:10.3917/rfs.491.0093. 


\section{eJRIEPS 47 Juillet 2020}

Gasparini, A., \& Pardo, A. (2000). De l'école au collège. Analyse des changements et des prolongements du mode de scolarisation scolaire. (Rapport de recherche. Groupe de Recherche sur la Socialisation). Lyon : Université Lyon 2.

Glaser, B., \& Strauss, A. (2010). La découverte de la théorisation ancrée : stratégies pour la recherche qualitative, (traduit par P. Paillé). Paris: Armand Colin.

Guigue, M. (2014). Ethnographies de l'école. Une pluralité d'acteurs en interaction. Bruxelles : De Boeck.

Heinich, N. (2004). Retour sur la notion d'élite. Cahiers internationaux de sociologie, 117(2), 313-326. doi:10.3917/cis.117.0313.

Jacobs, M. (2016). "So you're doing a research about black students ? Ethnographie dans deux écoles de Johannesbourg ». Dans J-P. Payet (dir.), Ethnographie de l'école, les coulisses des institutions scolaires et socio-éducatives, 99-116). Rennes : PUR.

Kherroubi, M., \& Rochex, J.-Y. (2004). La recherche en éducation et les ZEP en France. 2. Apprentissages et exercice professionnel en ZEP: résultats, analyses, interprétations, Revue française de pédagogie, 146, 115-190.

Lahire, B. (1998). L'homme pluriel. Paris : Nathan

Marsault, C. (2005). Les programmations en EPS : la mise en forme des APS. STAPS, 1(67), 9-22. Doi : 10.3917/sta.067.0009

Marsenach, J. (1989). Les pratiques des enseignants d'éducation physique et sportive dans les collèges. Revue française de pédagogie, 89, 7-10.

Mennesson C., \& Juhle S. (2012). "L'art (tout) contre le sport ? ». La socialisation culturelle des enfants des milieux favorisés ». Politix, 3, (99), 109-128.

Meuret, D. (2011). Elite scolaire et démocratie, Le Télémaque. 39(1), 41-52. doi:10.3917/tele.039.0041.

Meuret, D. (1995). Distribution sociale des facteurs d'efficacité des collèges, dans J.-M. Besse et al., Ecole efficace : de l'école primaire à l'Université (p. 81-91). Paris : Armand Colin.

Parlebas, P. (1995). La mise en ordre sportive. Sport, relations sociales et action collective. Bordeaux : MSHA.

Parlebas, P. (1986). Éléments de sociologie du sport. Paris : Presses universitaires de France.

Pasquier, D. (2005). Cultures lycéennes : La tyrannie de la majorité. Paris : Autrement. 


\section{eJRIEPS 47 Juillet 2020}

Paty, D. (1981). Douze collèges en France, le fonctionnement réel des collèges publics. Paris : La documentation française.

Payet, J-P. (2016). Observer les mondes scolaires disqualifiés. Dans JP. Payet (dir.) Ethnographie de l'école: Les coulisses des institutions scolaires et socioéducatives (pp.59-78). Rennes: Presses universitaires de Rennes.

Perrenoud, Ph. (1994). Métier d'élève et sens du travail scolaire. Paris : ESF.

Pétonnet, C. (1982). L'Observation flottante. L'exemple d'un cimetière parisien. L'Homme, tome $22, n^{\circ} 4$, Etudes d'anthropologie urbaine, 37-47.

Pinçon-Charlot, M., \& Pinçon M. (2007). Sociologie de la bourgeoisie. Paris : La découverte. Pinçon-Charlot, M., \& Pinçon M. (2014). Sociologie de Paris. Paris : La découverte.

Poggi, M. P. (2018). Introduction. Dans F. Brière-Guenoun, S. Couchot-Schiex, M-P. Poggi et I. Verscheure (dir.), Les inégalités d'accès aux savoirs se construisent aussi en EPS... (pp. 95-97). Université de Franche-Comté : Presses Universitaires de Franche-Comté.

Poggi, M. P. (2012). Recherches en EPS : tentative de problématisation sociologique. Vers une approche socio-didactique. Note de synthèse de l'Habilitation à Diriger des Recherches non publiée. Université de Franche-Comté.

Poggi, M. P. (2007). Le modèle sportif : une stratégie de recours en établissement défavorisé. Movement and Sport Sciences, 2(61), 59-71.

Poggi, M. P. (2002). Distribution des contenus d'enseignement en EPS au collège selon les caractéristiques, sociales du public scolaire : des différences non aléatoires. Revue française de pédagogie, 139, 53-69.

Poggi, M. P., \& Brière-Guenoun, F. (2014). « Ce qui s'enseigne en milieu difficile : tentative d'articulation des approches sociologique et didactique ». In N. Wallian, M.-P. Poggi , M. P., \& A. Chauvin-Vileno (dir.), Action, interaction, intervention : à la croisée du langage, de la pratique et des savoirs (pp. 333-364). Berne : Peter Lang.

Poggi, M. P., Verscheure, I., Musard, M., \& Lenzen, B. (2010). « Vers une approche sociodidactique en intervention ». Dans M. Musard, M. Loquet et Guy Carlier (dir). Sciences de l'intervention en EPS et sport. Résultats de recherche et fondements théoriques. Paris : EPS et ARIS.

Queval, I. (2008). Le corps aujourd'hui. Paris : Gallimard.

Rhein C., Le Pape A., \& Grosbras P-A. (1999). Division sociale de l'espace et inégalités de scolarisation, Rapport final de recherche au Plan Construction-Architecture, ministère de l'Équipement et du Logement. 


\section{eJRIEPS 47 Juillet 2020}

Sébileau, A. (2011). Le monopole professoral en question. Le cas de l'Education Physique et Sportive en France. ARSS, 3, 70-83.

Sensevy, G. (2002). Des catégories pour l'analyse comparée de l'action du professeur : un essai de mise à l'épreuve. In P. Venturini, C. Amade-Escot et A. Terrisse (Eds), Etude des Pratiques Effectives : l'approche des didactiques (pp. 25-47). Grenoble : La Pensée Sauvage.

Sensevy, G., \& Mercier, A. (2007). Agir ensemble: l'action didactique conjointe $d u$ professeur et des élèves. Rennes : Presses universitaire de Rennes.

Strauss, A. (1992). La trame de la négociation (textes réunis et présentés par Baszanger I.). Paris : L'Harmattan, coll. Logiques sociales.

Van Zanten, A. (2001). L'école de la Périphérie, scolarité et ségrégation en banlieue. Paris : Puf.

Vigneron, C. (2006). Les inégalités de réussite en EPS entre filles et garçons : déterminisme biologique ou fabrication scolaire ?, Revue française de pédagogie, 154, 111-124.

Vors, O. (2013). Une leçon avec une classe difficile: des stratégies d'intervention adaptées à trois périodes typiques. Dans B. Boda et A. Coston (Eds.), La leçon d'EPS en questions (p. 44-47). Paris : AEEPS.

Wilson, A.B. (1959). Residential Segregation of Social Classes and Aspirations of High School Boys. American Sociological Review, 24, 836-845.

Zolesio, E. (2010). Dispositions féminines/dispositions masculines ", Revue $i$ Interrogations ?,10. http://www.revue-interrogations.org (Consulté le 8 février 2019). 